\title{
Effectiveness of Acupuncture as Adjunctive Therapy in Type 2 Diabetic: Study Protocol for a Randomized Controlled Trial
}

\section{Yean Chin Cheok}

Universiti Putra Malaysia Fakulti Perubatan dan Sains Kesihatan https://orcid.org/0000-0002-48531332

\section{Zalilah Mohd Shariff ( $\nabla$ zalilahms@upm.edu.my)}

Universiti Putra Malaysia Fakulti Perubatan dan Sains Kesihatan

\section{Chan Yoke Mun}

Universiti Putra Malaysia Fakulti Perhutanan dan Alam Sekitar

\section{Lee Ping Yein}

Universiti Putra Malaysia Fakulti Perubatan dan Sains Kesihatan

\section{Ng Ooi Chuan}

Universiti Putra Malaysia Fakulti Perubatan dan Sains Kesihatan

\section{Research Article}

Keywords: Type 2 diabetes mellitus, Acupuncture, Randomized Controlled Trial

Posted Date: January 3rd, 2022

DOl: https://doi.org/10.21203/rs.3.rs-718907/v1

License: (c) (i) This work is licensed under a Creative Commons Attribution 4.0 International License. Read Full License 


\section{Abstract}

Background: The incidence of type 2 diabetes mellitus is increasing worldwide. The literature suggests that acupuncture is a possible complementary therapy for type 2 diabetes mellitus. This study aims to determine the effect of acupuncture as an adjunctive therapy on homeostasis model assessment-insulin resistance (HOMA-IR), adiposity and health-related quality of life (HRQOL) in patients with type 2 diabetes mellitus.

Method and design: This randomised, double-blind, placebo controlled and parallel design trial will be carried out in three public university teaching hospitals in Malaysia. Eligible type 2 diabetes mellitus subjects will be randomly assigned to receive either acupuncture $(n=30)$ or a placebo $(n=30)$. The intervention is carried out using acupuncture 'press' needle on abdomen area (10 sessions of treatment), twice a week over 6 weeks. Both groups will continue with their routine diabetes care. Primary outcome of HOMA-IR and secondary outcomes of body mass index and waist circumference will be measured at the time of recruitment (baseline) and after completion of 10 sessions (week 7) of the treatment. Additionally, secondary outcome of HRQOL will be measured at the time of recruitment (baseline), after completion of 5 sessions (week 3/4) and 10 sessions (week 7) of the treatment. Any adverse event will be recorded at every visit.

Discussion: The findings of this study will provide important clinical evidence for the effect of acupuncture as adjunctive therapy on HOMA-IR, adiposity and HRQOL of type 2 diabetes mellitus.

Name of the registry: US National Institutes of Health Clinical Trials Registry (ClinicalTrials.gov).

Trial registration Number. NCT04829045

Date of registration: 2 April 2021

URL of trial registry record: https://clinicaltrials.gov/ct2/show/NCT04829045?

cond=Type+2+diabetes\%2C+acupuncture\&draw $=2 \&$ rank $=1$

\section{Background}

Globally, approximately 463 million people are living with diabetes mellitus (DM)[1] with most are type 2 DM $[2,3]$. Type 2 DM involves a decline in b-cell function and increase in insulin resistance $[4,5]$. Long term insulin resistance is associated with many complications $[6,7]$ which will adversely affect quality of life [8]. Currently, among the several mechanism-based drugs available for treatment of type 2 DM, only insulin sensitizers such as biguanides and thiazolidinediones have direct effects on insulin resistance [9, 10]. However, pharmacological agents have limitations related to patient's poor adherence or resistance to intensify treatment and clinical inertia $[5,11]$. These may be associated with the fear of side-effects of the medications [11]. Complementary and alternative medicine (CAM) may provide a beneficial adjunctive therapy for DM due to its perceived less adverse effect and culturally more acceptable in Asian 
population. Among the CAM, acupuncture is one of the most widely used combined therapies with conventional treatment for type 2 DM [12]. Additionally, the United States National Institutes of Health $(\mathrm{NIH})$ has acknowledged that acupuncture can be used as adjunctive treatment for DM [13] with minimum adverse effect $[14,15]$ by a well-trained practitioner [16].

Acupuncture is a technique involving the insertion of very fine needles into specific points to affect the flow of body's qi [17], or vital energy. According to the theory of traditional Chinese medicine (TCM), acupuncture can regulate qi and blood and have a direct impact on bioavailability of substances of the body [18]. In the past 20 years, several randomized controlled trials (RCTs) have been conducted on the effectiveness of acupuncture on DM and its complications [19-21]. It has been demonstrated that acupuncture may improve insulin sensitivity $[18,22]$ and c-peptide levels through effective treatment against metabolic disturbances such as hyperglycemia [21, 23, 24], overweight [24, 25], inflammation [26], while improving lipid metabolism [27]. Equally, systematic reviews have also confirmed the benefits of acupuncture in patients with DM $[28,29,30]$. On the other hand, acupuncture is able to control comorbidity conditions such as obesity [31, 32], pain [33,34], anxiety, depression [35], stress [36], sleeping disorder $[37,38]$ and hence improves quality of life $[39,40]$. However, multiple methodological flaws with substantial risk of bias have been identified in many of these RCTs [40,41]. Therefore, this double-blind, placebo controlled clinical trial is designed to investigate the effect of acupuncture as adjuvant therapy in patients with type 2 DM in accordance with the consolidated Standards for Reporting of Trials Statement 2010 (CONSORT 2010)[42] and revised Standards for Reporting Interventions Controlled Trials of Acupuncture 2010 (STRICTA 2010) [ 43] guidelines.

\section{Methods/design}

\section{Objectives}

This study aims to determine the effect of acupuncture treatments on homeostasis model assessmentinsulin resistance (HOMA-IR), adiposity and health-related quality of life (HRQoL) in patients with type 2 DM.

\section{Trial design}

This is a randomized, double-blinded (patients and practitioners), placebo and two-arm parallel controlled clinical trial. This study will be conducted at three public university teaching hospitals in Malaysia. The reporting of the trial will adhere to the CONSORT [42] and STRICRTA [43] guidelines.

\section{Ethics approval}

The trial protocol has been approved by the Universiti Putra Malaysia Ethics Committee for Research Involving Human Subjects (JKEUPM-2018-294) and registered in ClinicalTrial.gov (NCT04829045). The study is performed according to Declaration of Helsinki and the guidelines for Good Clinical Practice. 
Permission to carry out the study will be obtained from the respective hospitals before conducting the research.

\section{Sample-size calculation}

A total of 30 patients are required in each group to detect a significant mean difference of $1.23 \% \mathrm{HOMA}-$ IR, $1.25 \%$ standard deviation [18], 95\% confidence level and $80 \%$ power, with an additional dropout rate of $30 \%$.

\section{Methods of recruitment}

Subject recruitment is conducted at public university teaching hospitals in Malaysia by an endocrinologist and a TCM practitioner through patients' routine medical check-up schedules. Patients will be provided study brochure and information sheet if they express interest to participate in the study. Upon agreement to participate in the study, patients will be requested to fill up a screening form to assess their study eligibility. For screening purpose, type $2 \mathrm{DM}$ is defined as one abnormal fasting plasma glucose (FPG) $\geq 7.0 \mathrm{mmol} / \mathrm{L}(126 \mathrm{mg} / \mathrm{dL})$, random venous plasma glucose $\geq 11.0 \mathrm{mmol} / \mathrm{L}(200 \mathrm{mg} / \mathrm{dL})$, two hour plasma glucose measurement $\geq 11.0 \mathrm{mmol} / \mathrm{L}(200 \mathrm{mg} / \mathrm{dL})$ or glycated hemoglobin $(\mathrm{HbA} 1 \mathrm{c}) \geq$ $6.5 \%$ based on subjects' self-reported or through their DM book, medications packages, and clinic follows up cards. Those who are eligible will be invited to attend a blood collection session. Type $2 \mathrm{DM}$ is confirmed with subject's HbA1c $\geq 6.5 \%$ or morning FPG $\geq 7.0 \mathrm{mmol} / \mathrm{L}(126 \mathrm{mg} / \mathrm{dL})$ (fasting at least 10 hours) based on American Diabetes Association recommendation [44]. Patients are required to sign a written informed consent form once they fulfill all the study criteria and agree to further participate in the study.

\section{Eligibility criteria}

The subject eligibility criteria for the study include Malaysian, age between 30-69 years old, have had type $2 \mathrm{DM}$ (FPG $\geq 7.0 \mathrm{mmol} / \mathrm{L}[126 \mathrm{mg} / \mathrm{dL}]$ or $\mathrm{HbA} 1 \mathrm{c} \geq 6.5 \%$.) for more than one year and with $\mathrm{BMI} \leq$ $40.0 \mathrm{~kg} / \mathrm{m}^{2}$; receive oral anti-diabetic agents on a stable dose over the previous 3 months. Patients with any of the following criteria will be excluded: 1 ) under insulin therapy; 2) with other acute or chronic health problems; 3 ) needle phobia or allergy to adhesive plaster; 4) planning to move out from Malaysia within 4 months' and 5) being pregnant, planning for pregnancy or lactating women.

\section{Randomization and allocation strategy}

Blocked randomisation with block size of 2 will be performed in a 1:1 allocation ratio to minimise between-group differences on HOMA-IR level and body mass index (BMI). The press needles and placebos are identical in appearance. They are repacked in identical plastic containers and consecutively numbered for each subject according to the randomization schedule. Each subject is assigned an order number. The containers will be distributed to the principle investigator at the sites and practitioner(s) will receive the corresponding containers which contain either press needles or placebos. Randomization sequence and allocation will be concealed to all subjects, acupuncturists, laboratory personnel and 
partial researchers. Sequentially numbered, sealed opaque envelopes will be used to conceal treatment procedures. The randomization sequence will remain concealed until the end of the study.

\section{Compliance}

Adherence to the protocol is assessed at every follow-up session and evaluation of intervention adherence will be assessed at post intervention.

\section{Intervention}

Subjects will receive 10 sessions of real or placebo acupuncture treatment using Pyonex Press Needles or Pyonex Placebos. During the treatments, all subjects are required to take supine position. New, disposable, sterilized, stainless-steel press needles are utilized. The acupuncture points will be disinfected with a disposable $70 \%$ isopropyl alcohol cotton. After all the press needles are fixed, acupoints are pressed for at least three times (at 0,15 and after 30 minutes) for about 30 seconds, with the degree that can be tolerated by the subject. The press needles and placebos are inserted according to the acupoints selection sequence, the product directions and following same procedures [45]. The validity and credibility of the device have been well demonstrated in other studies $[46,47]$.

In this study, an irradiating needling sensation (de qi) does not have to be achieved. "De qi" is a tingling, numbness, and heaviness feelings that occur after an acupuncture needle had been inserted in the body [48]. During the 45 minutes needle retention period, subject's abdomen will be covered to prevent exposure. If the subjects are sick or not felling well on that day, they will not be given any treatment and are requested to come on other day. Similarly, if subject reports discomfort or encounter any adverse effect during intervention, acupuncture therapy is discontinued and the treatment for that particular session will not be included.

The study period is seven weeks with intervention period for 6 weeks and follow-up for any adverse event for 1 week. Treatments are performed twice per week. However, in certain circumstances, once-a-week treatment to a three-time per week treatment is permitted, but ten treatments must be completed within six weeks. For those who are under drug(s) or other treatments during intervention, they are required to continue with their existing treatment regimens. All medication and doses received by the subjects are recorded and monitored during the course of the study to ensure the medications are maintained throughout the study. Medications that are allowed in this study are oral anti-diabetic drugs, including hypertensive and hyperlipidemia medications. Nevertheless, medications that are not allowed in this study are all types of insulin; Glucagon-like peptide-1 receptor agonists such as Liraglutide, Exenatide, Dulaglutide, Semaglutide and thiazolidinediones (TZDs) such as Pioglitazone and Rosiglitazone. There will be no change in other treatment as long as patients are clinically stable. Subjects are also advised to notify the practitioners immediately if there are any changes in their current treatments/circumstances such as personal details, medication or pregnancy (for woman) during the study. Any changes in the treatment regimen will be documented. Subject's is considered drop-outs if they have missed their appointments for more than three times, they do not attend the baseline or assessment session (cause 
missing data), they become pregnant (for women), inability to comply with protocol requirements, withdrawal of his/her own decision or advised by the researcher (for example participant increase $20 \%$ doses of DM medication or using acupuncture at other clinic during intervention). Safety or adverse event in each session will also be recorded in a case record form. Data will be collected and evaluated at the time of recruitment (baseline), after completion of 5 sessions (week 3/4) and 10 sessions (week 7) of the treatment.

\section{Treatment group}

The treatment group will receive real acupuncture treatments using Pyonex Press Needles (Pyonex $\varnothing 0.20 \times 1.5 \mathrm{~mm}$ made by Seirin Corporation). A standardized treatment with 6-10 needles on abdomen area such as Zhongwan (Ren-12), Xiawan (Ren-10), Qihai (Ren-6), Guanyuan (Ren-4), Tian Shu (St-25), Daheng (Sp-15), Shangqu, Jinhe (M-HN)/Qixue (Kd-13), Shuidao (St-28) and Liang Men (St-21) are selected based on literature reviews $[18,22,49,50]$ and recommendation by international experts. The number and name of acupuncture points used in each session will be documented.

\section{Control group}

The same procedure and acupuncture points are used for the control group. Subject are given placebo which is identical to the press needle except the needle element has been removed and replaced with a blunt knob in all aspects are indistinguishable to the press needles [51].

\section{Practitioners' background and training}

Interventions are administered by 3 qualified TCM practitioners or acupuncturists with at least five years of clinical experience and have had degree academic qualification in relevant field. They are required to undergo an intensive training to ensure consistency of the intervention. A standard operation procedure will be provided during the training such as identification of the acupoint location, compulsory acupoints to be used in each session, technique in performing the study treatment and the time to press needles after insertion of the needles. They will also be briefed about risk management of the trial during the training. To ensure the practitioners are masked to the group allocation, practitioners are requested not to check for the presence of a sharp tip below the plaster during the intervention.

\section{Measurements}

Socio-demographic backgrounds of patients which include patients' age, gender, ethnicity, marital status, educational level, personal monthly income, duration of DM, and family history of DM will be obtained through a questionnaire at recruitment. The primary and secondary outcomes will be measured at baseline, during and after the treatment is completed. Table 1 shows the schedule for data collection during the 7 weeks of study period. Data will be collected at the time of recruitment (baseline), after completion of 5 sessions (week 3/4) and 10 sessions (week 7) of the treatment.

\section{Primary outcome}


The primary outcome of this trial is the change in HOMA-IR after treatment. Fasting insulin and glucose concentrations will be used to derive the HOMA-IR [fasting serum insulin $(\mu \mathrm{U} / \mathrm{ml}) \times$ fasting plasma glucose (mmol l-1)/22.5][52]. Fasting insulin levels and fasting glucose will be analyzed using heparin tubes and sodium fluoride tubes respectively by an enzyme-linked immunosorbent assay, also called ELISA or EIA. Peripheral blood will be drawn from the antecubital veins of the subjects at baseline and at week 7 after a 10 hour overnight fasting. A total of $10 \mathrm{ml} / \mathrm{L}$ of venous blood will be obtained using venipuncture method. These blood samples are then centrifuged for 10 minutes at the speed of $35000 \mathrm{rpm}$ to separate the blood serum. Subsequently, serum will be put in an Eppendorf tubes for preservation. The serum is preserved in a low temperature freezer which shall maintain at a temperature of -70 Celsius.

\section{Secondary outcomes}

The secondary outcomes are changes in health-related quality of life (HRQoL) and anthropometry parameters such as weight, body mass index (BMI) and waist circumference (WC). Subject's HRQoL will be assessed using a validated World Health Organization Quality of Life Assessment: Brief Version (WHOQoL-BREF [English/Malay]) questionnaire [53,54]. Anthropometry measurements will be measured according to the standard procedure. Subjects will be measured for weight in kilogram to the nearest 0.1 kilogram, using a digital scale TANITA model HD-382 weighing machine in a standing position. Height will be measured using SECA body meter model 208 to the nearest 0.05 centimetre. $\mathrm{BMI}$ is calculated weight in kilogram divided by height in meters squared (BMI=Weight $(\mathrm{kg}) /$ Height $^{2}\left(\mathrm{~m}^{2}\right)$. WC will be measured midway between the lowest rib and the iliac crest using SECA measuring tape to the nearest 0.05 centimetre. Central obesity for Asian population is defined as having WC $\geq 90 \mathrm{~cm}$ for men and $\geq 80 \mathrm{~cm}$ for women based on WHO/IASO/IOTF criteria [55].

\section{Statistical analysis}

Data will be analyzed using SPSS Statistics 26.0 (IBM Corp., New York, USA). Kolmogoro-Smirnov tests for normality will be performed on each variable prior to analysis. Comparisons of baseline continuous variables between intervention and control group will be examined using independent $t$ test, while for categorical variables the chi-square test will be used. Paired $t$ test will be used for within group differences. Intention-to-treat analysis (ITT) will be performed, and all patients enrolled will be included in the analysis, irrespective of compliance. Missing data is imputed using last observation carried forward method. The GLM procedure for repeated-measures ANCOVA will be performed to test the main effect of within-subject and between-subject factors as well as the interaction of these factors while controlling for possible baseline covariate. The significant level for all tests is set at $p<0.05$ (two-tailed).

\section{Adverse events}

Safety or adverse event suspected related to the treatment, through the symptoms reported by the patients, and observations by researcher at every visit are collected. Adverse event is defined as any unfavourable and unintended sign, symptom, or disease temporally associated with the use of a medical treatment or procedure that may or may not be considered related to the medical treatment or procedure 
[48]. Possible adverse events related to acupuncture are bruising, nausea, temporary pain, dizziness or faintness, or discomfort [56]. Details about the event time, severity, and duration, causal relationship with the treatment, other treatments or medications that are suspected to cause the adverse event will be recorded.

\section{Discussion}

DM is increasing rapidly globally, particularly in low-and middle-income countries and has been listed as one of the top 10 causes of premature mortality in Malaysia [57]. While type 2 DM is predominant in middle-aged and elderly people previously, it is now increasingly prevalent in children and young people. For instance, adults aged $\geq 30$ years with type 2 DM in Malaysia has been increasing, affecting 2.8 million individuals [58]. Overweight is one of the risk factors of type $2 \mathrm{DM}$. Obese people have high levels of non-esterified fatty, acids, cytokines, glycerol, pro-inflammatory markers, hormones and other substances that are actively involved in development of insulin resistance [59]. In 2019, it was revealed that one in two adults in Malaysia was overweight, obese or with abdominal obesity [60]. Therefore, the increasing trend of overweight to a concomitant rise of type 2 DM is expected in Malaysia. Several studies have concluded that acupuncture may improve insulin sensitivity $[22,27]$ and is effective against metabolic disturbances including obesity [32].

The mechanism by which acupuncture helps to reduce obesity and insulin resistance is unclear. However, studies suggested that acupuncture increases the neural activity associated with the ventromedial nuclei of the hypothalamus; raises the tone of the smooth muscle of the stomach and decreases circulating leptin and insulin levels [61]; reduce body weight by reducing food intake and inducing lipid metabolism; lowers blood glucose level by inducing insulin secretion; improves insulin signal pathway by activating phosphorylaltlion-3 kinase (P13K)-mediated activation of/Akt and inducing expression of insulin signal proteins (IRSI/GLUT4) in insulin-sensitive organs; ameliorates inflammation by depressing hyperactivity of sympathetic nervous system (SNS) and regulating vagus nerve activity [27]. Hence, it is pertinent to determine the treatment effect of acupuncture which may offer more insights on the prevention and management of patients with type $2 \mathrm{DM}$.

Currently, it was estimated that the annual global health care spending on DM was US\$ 850 billion in 2017 [62]. It is a significant challenge for healthcare systems with such a high cost. Acupuncture may provide beneficial adjunctive therapy for type 2 DM due to its cost effectiveness [63-65]. For instance, study conducted on pelvic and low back pain in pregnancy (TAPP) in the France shown that costeffectiveness of acupuncture was higher as compared to conventional medical treatments. Average total costs were higher in the control group (€2947) than in the acupuncture group (€2635), with a difference of 312 Euros (95\%Cl:€-966;€325)[66]. From a Western academic perspective, acupuncture appears to be a cost-effective intervention for pain established by the UK's National Institute for Clinical Excellence [67].

Acupuncture is a new treatment modality, emerging within the last two decades. To the best of our knowledge, currently there are limited well-designed RCTs to determine the efficacy of acupuncture on 
type 2 DM. In Southeast Asia, two acupuncture clinical trials on type 2 DM had been carried out; one is at Indonesia and another one is at India $[23,68]$. Safety is one of the most important issues in the use of acupuncture in clinical practice, especially for DM. Nevertheless, according to various studies in China, Korea, Japan, Germany and the United Kingdom, serious adverse event associated with acupuncture is rare $[14,15,69]$ where the incidence rate is about $0.004 \%$. Treatment with shallow insertion using press needles have been described and recommended [47, 51]. It is generally more practical, safe and scientifically approved as they are away from internal organ. Short needle (length $1.5 \mathrm{~mm}$ ) with shallow needling can reduce the side effects of pain, traumatic, bleeding or haematoma during intervention [51].

\section{Conclusion}

This randomized controlled trial will contribute to the gap in knowledge on the effectiveness of acupuncture as adjunctive therapy in patients with type 2 DM based on the previous research and practical significance. It is expected that subjects in the intervention group will show improvements in HOMA-IR levels and/or HRQoL as compared to their counterparts. The findings of this study can be used to develop evidence-based recommendations of acupuncture as adjunct therapy on HOMA-IR and/or HRQoL of type 2 DM patients, and will contribute to our understanding of the mechanisms of acupuncture in relation to type $2 \mathrm{DM}$.

\section{Declarations}

\section{Trial Status}

Protocol version Issue date: 11 July 2021

Authors: Cheok Yean Chin , Zalilah Mohd Shariff, Chan Yoke Mun, Lee Ping Yein and Ng Ooi Chuan

Date of recruitment: $27^{\text {th }}$ May $2021-26^{\text {th }}$ May 2022

\section{Ethics approval and consent to participate}

The trial protocol has been approved by the Universiti Putra Malaysia Ethics Committee for Research Involving Human Subjects (JKEUPM-2018-294) and patients are required to sign a written informed consent form before participating in the study.

\section{Consent for publication}

Not applicable

\section{Availability of data and material}

Not applicable

Competing interests 
The authors declare that they have no competing interests.

\section{Funding}

This study is sponsored by the Universiti Putra Malaysia and acupuncture needles are contributed by SEIRIN Corporation, Japan. An application for funding to support clinical trial costs has been made to Hai-O Enterprise, Malaysia.

\section{Authors' contributions}

Cheok Yean Chin, Zalilah Mohd Shariff, Chan Yoke Mun and Lee Ping Yein are responsible for the preparation of the study protocol, case record forms (CRF) and relevant documents with $\mathrm{Ng}$ Ooi Chuan provided inputs for revision. All investigators will oversee the trial implementation. Cheok Yean Chin and $\mathrm{Ng}$ Ooi Chuan will assist in recruiting patients, performing, and overseeing treatment at various study sites. All investigators will contribute to any report / publication outcomes of this trial.

\section{Acknowledgements}

Not applicable

\section{References}

1. International Diabetes Federation. IDF Diabetes Atlas, 9th edn. Brussels, Belgium: 2019. https://www.diabetesatlas.org. Accessed 19 October 2020.

2. World Health Organization (2019). Classification of diabetes mellitus 2019. Licence: CC BY-NC-SA 3.0 IGO. Accessed 12 July 2019.

3. American Diabetes Association (ADA). (2016). Diabetes Care, standards of medical care in diabetes2016. Vol 39, Sup. 1.

4. Olokoba, Obateru \& Olokoba. Type 2 diabetes mellitus: a review of current trends. Oman Medical Journal. 2012; 27(4): 289-273. DOI 10. 5001/omj.2012.68

5. Reach G, Pechtner V, Gentilella R, Corcos A and Ceriello, A. Clinical inertia and its impact on treatment intensification in people with type 2 diabetes mellitus. Diabetes \& Metabolism. 2017;501-511.

6. María M. Andany A, Calder RF, Fernandez CF, Rodríguez EA, Montero AD. Subclinical vascular disease in patients with diabetes is associated with insulin resistance. Diabetes \& Metabolic Syndrome: Clinical Research \& Reviews. 2019;13: 2198-2206.

7. Ekblad LL, Rinne JO, Puukka P, Laine H, Ahtiluoto S, Sulkava R, Vitanen M and Jula A. Insulin resistance predicts cognitive decline: an 11-year follow-up of a nationally representative adult population sample. Diabetes Care. 2017;40: 1-8.

8. Trikkalinou A, Papazafiropoulou AK and Melidonis A. Type 2 diabetes and quality of life. World Journal of Diabetes. 2017;8(4): 120-129. 
9. Olefsky JM and Saltiel AR. "PPARy and the treatment of insulin resistance". Trends in Endocrinology and Metabolism. 2000,11(9): 362-368.

10. Fonseca V, Desouza C, Asnani S, Jialal I. Nontraditional risk factors for cardiovascular disease in diabetes. Endocr Rev. 2004;25: 153-175.

11. Kassahun A, Gashe F, Mulisa E and Rike WA. Nonadherence and factors affecting adherence of diabetic patients to anti-diabetic medication in Assela General Hospital, Oromia Region, Ethiopia. J Pharm Bioallied Sci. 2016;8: 124-129. doi: 10.4103/0975-7406.171696.

12. Chen C, Liu J, Sun M, Liu W, Han J, Wang H. Acupuncture for type 2 diabetes mellitus: A systematic review and meta-analysis of randomized controlled trials. Complement Ther Clin Pract. 2019;36:100112. doi: 10.1016/j.ctcp.2019.04.004. Epub 2019 Jul 2. PMID: 31383426.

13. Takahashi H. Effects of Acupuncture on Terminal Cancer Patients in the Home Care Setting. Medical Acupuncture. 2009;21(2): 123-129.

14. Witt $C M$, Pach $D$, Reinhold T et al. Treatment of the adverse effects from acupuncture and their economic impact: A prospective study in 73,406 patients with low back or neck pain. Eur J Pain. 2011;15:193-197.

15. Birch S, Alraek T and Norheim AJ. The Journal of Alternative and Complementary Medicine. 2013;19: 845-850. DOI: $10.1089 / \mathrm{acm} .2012 .0639$.

16. MacPherson $\mathrm{H}$, Hammerschlag R, Lewith $\mathrm{G}$ and Schnyer R. Acupuncture Researh, Strateies for Estalishing an Evidence Base. Churchill Livingstone, 2008.

17. British Acupuncture Council. Acupuncture; 2021. https://acupuncture.org.uk/aboutacupuncture/what-is-acupuncture/. Accessed 12 July 2021.

18. Firoujaei A, Li GC, Wang N, Liu WX and Zhu BM. Comparative evaluation of the therapeutic effect of metformin monotherapy with metformin and acupuncture combined therapy on weight loss and insulin sensitivity in diabetic patients. Nutrition \& Diabetes. 2016;6, e209; doi: 10.1038/nutd2016.16.

19. Shen PF, Kong L. [Effects of acupuncture on mood and glucose metabolism in the patient of type 2 diabetes]. Zhongguo Zhen Jiu. 2007;27:741-3.

20. Zhang ZL, Ji XQ, Zhao SH, Zhang JJ, Kang T, Yang XJ. [Randomized controlled study on effects of the needling method for regulating spleen-stomach on coronary heart disease complicated by type 2 diabetes mellitus complicated]. Zhongguo Zhen Jiu. 2008;28:629-33.

21. Peplow PV, Baxter GD. Electroacupuncture for control of blood glucose in diabetes: literature review. J Acupunct Meridian Study. 2012;5:1-10.

22. Lin RT, Pai HC, Lee YC, Tzeng CY, Chang CH, Hung PH, Chen Yl, Hsu TH, Tsai CC, Lin JG and Chang SL (2013). Electroacupuncture and rosiglitazone combined therapy as a means of treating insulin resistance and type 2 diabetes mellitus: a randomized controlled trial. Evidence-Based Complementary and Alternative Medicine. 2013:969824. doi: 10.1155/2013/969824.

23. Tjipto BW, Saputra K, Sutrisno TC. Effectiveness of acupuncture as an adjunctive for diabetes mellitus: A randomized controlled trial. Medical Acupuncture. 2014;26: https://doi.org/10.1089/acu.2014.1058. 
24. Tur FC, E Kilic TY and Temizyurek Z. Therapeutic effects of acupuncture on obesity and HbA1c. European Journal of Integrative Medicine. 2014;7: 88-93.

25. Zhang K, Zhou S, Wang C, Xu H \& Zhang L. Acupuncture on obesity: clinical evidence and possible neuroendocrine mechanisms. Evidence-Based Complementary and Alternative Medicine. 2018;6409389: 1-15. https://doi.org/10.1155/2018/6409389N

26. Wei $\mathrm{Y}$, Dong $\mathrm{M}$, Zhang $\mathrm{H}$ et al. Acupuncture attenuated inflammation and inhibited Th17 and treg activity in experimental asthma. Evidence-Based Complementary and Alternative Medicine. 2015; Article ID 340126. http://dx.doi.org/10.1155/2015/340126

27. Liang F and Koya D. Acupuncture: is it effective for treatment of insulin resistance. Diabetes. Obesity and Metabolism. 2009;2: 555-569.

28. Cai and Peng (2010). 'Meta analysis on acupuncture treatment of diabetes'. Chinese Archives of Traditional Chinese Medicine. 28,(11):2412-2415.

29. Yang MX, Li XM, Liu SH. Meta-analysis of acupuncture for relieving non-organic dyspeptic symptoms suggestive of diabetic gastroparesis. BMC complementary and alternative medicine 2013;13(1): 311.

30. Dimitrova A, Murchison C, Oken B. Acupuncture for the Treatment of Peripheral Neuropathy: A Systematic Review and Meta-Analysis. J Altern Complement Med. 2017;23(3):164-179. doi:10.1089/acm.2016.0155.

31. Zhang RQ, Tan J, Li FY, Ma YH, Han LX, Yang XL. Acupuncture for the treatment of obesity in adults: a systematic review and meta-analysis. Postgrad Med J. 2017;93(1106):743doi:10.1136/postgradmedj-2017-134969.

32. Zhong YM, Luo XC, Chen Y, et al. Acupuncture versus sham acupuncture for simple obesity: a systematic review and meta-analysis. Postgrad Med J. 2020;96(1134):221doi:10.1136/postgradmedj-2020-137221.

33. Manyanga T, Froese $M$, Zarychanski $R$, et al. Pain management with acupuncture in osteoarthritis: a systematic review and meta-analysis. BMC Complement Altern Med. 2014;14:312. doi:10.1186/1472-6882-14-312.

34. Helianthi DR, Simadibratai C, Srilestari A et al. Pain reduction after Laser Acupuncture treatment in geriatric patients with knee osteoarthritis: a randomized controlled trial. Acta Med Indon-Indon J Intern Med. 2016;117-121.

35. David P, Sniezek DC, FAAMA and Imran J. Siddiqui. Acupuncture for Treating Anxiety and Depression in Women: A Clinical Systematic Review. Medical Acupuncture. 2013;25(3): 164-172. doi: 10.1089/acu.2012.0900.

36. Mishra S, Archana R. Sailesh KS., Bhasetti S, Jose J, Reddy UK, Mukkadan JK. et al. Stress management through manual-acupuncture in type-2 diabetic patients. Journal of Chemical and Pharmaceutical Sciences. 2017;10(3): 1153-1155.

37. Cao H, Pan H, Liu J. Acupuncture for treatment of insomnia: a systematic review of randomized controlled trials. Journal of Alternative and Complementary Medicine. 2009; 15: 1171-1186. 
38. Dong B, Chen Z, Yin X et al. The efficacy of acupuncture for treating depression-related insomnia compared with a control group: a systematic review and meta-analysis. BioMed Research International. 2017; ID9614810, http://doi.org/10.1155/2017/96.

39. Vinjamury PS, Li JZ, Hsiao E, Huang C., Hawk C, Miller J and Huang Y. Effects of acupuncture for cancer pain and quality of life - a case series. BioMed Central. 2013;8: 15.

40. Xiong WJ, Feng X, Liu JP, Chen W. Electroacupuncture for treatment of diabetic peripheral neuropathy: a systematic review of randomized controlled trials. Journal of Traditional Chinese Medical Sciences. 2016;3:9-21.

41. Bo C, Xue Z, Yi G, Zelin C, Yang B, Zixu W and Yajun W. Assessing the quality of reports about randomized controlled trials of acupuncture treatment on diabetic peripheral neuropathy. PloS One. 2012;7(7): e38461.

42. Schulz KF, Altman DG, Moher D, for the CONSORT Group. 'CONSORT 2010 Statement: updated guidelines for reporting parallel group randomised trials'. BMJ. 2010; [online]. 340(c332).

43. MacPherson H, Altman DG, Hammerschlag R, Li Y, Wu T, White A, Moher D, on behalf of the STRICTA Revision Group. 'Revised Standards Reporting Interventions in Clinical Trials of Acupuncture (STRICTA): Extending the CONSORT Statement'. PLoS Medicine. 2010; [online]. 7(6).

44. Diabetes Care 2021 Jan; 44(Supplement 1): S1-S2. https://doi.org/10.2337/dc21-Sint. Accessed 3 July 2021.

45. Miyazaki S, Hagihara A, Kanda R, Mukaino Y. Nobutomo K. Applicability of press needles to a doubleblind trial: a randomized, double-blind, placebo-controlled trial. The Clinical Journal of Pain. 2009;25(5):438-444. DOI: 10.1097/AJP.0b013e318193a6e1.

46. Bai $Y$, Chen $S$, Chen $L$ and Wang B. Postoperative pain of mixed hemorrhoid treated by embedding needles in Erbai. World Journal of Acupuncture-Moxibustion (WJAM). 2015;25(1): 59-61.

47. Schroder S, Meyer-Hamme G, Friedemann T, Kirch S, Hauck M, Plaetke R, Friedrichs S, Gulati A \& Briem D. Immediate pain relief in adhesive capsulitis by acupuncture- a randomized controlled double-blinded study. Pain Medicine. 2017; 2235-2247. Doi: 10.1093/pmm/pnx052.

48. NCl, National Cancer Institute, Common Terminology Criteria for Adverse Events (CTCAE) v5.0. NIH. 2017. Accessed 6 Sept 2018.

49. Kim KW, Yoo HH, Cho et al. Effects of acupuncture on serum metabolic parameters in premenopausal obese women: study protocol for a randomized controlled trial. Trials. 2015;16: 327. DOI 10.1186/s13063-015-0867-y.

50. Zhong LD, Kun W, Lam TF et al. The combination effects of body acupuncture and auricular acupressure compared to sham acupuncture for body weight control: study protocol for a randomized controlled trial. Trials. 2016;17: 346. doi: 10.1186/S13063-016-1458-2.

51. Fleckenstein J, Baeumler PI, Gurschler C, Weissenbacher T, Simang M \& et al. Acupuncture for post anaesthetic recovery and postoperative pain: study protocol for a randomized controlled trial. Trials. 2014; 15: 292. 
52. Matthews DR, Hosker JP, Rudenski AS, Naylor BA, Treacher DF, Turner RC. Homeostasis model assessment: Insulin resistance and beta-cell function from fasting plasma glucose and insulin concentrations in man. Diabetologia. 1985;28:412-9.

53. World Health Organization's Quality of Life group: WHOQOL-BREF Introduction, Administration and Scoring (1998), Field Trial version.

54. Hasanah $\mathrm{Cl}$, Naing L and Rahman ARA. World Health Organization Quality of Life Assessment: Brief Version in Bahasa Malaysia. Med J Malaysia. 2003; 58(1): 79-88.

55. World Health Organization. Regional Office for the Western Pacific. (2000). The Asia-Pacific perspective: redefining obesity and its treatment. Sydney: Health Communications Australia. https://apps.who.int/iris/handle/10665/206936. Accessed 12 July 2021.

56. Hempel S, Taylor SL, Solloway M, MIAKE-Lye IM, Beroes JM, Shanman R, Booth MJ, Siroka AM, Shekelle PG. Evidence map of acupuncture. VA-ESP Project \#05-226; 2013.

57. Ummi NY, Diana M, Azahadi O et al. Burden of premature mortality in Malaysia. Int J Public Health Res. 2013; 3: 249-56.

58. Zanariah H, Sri WT, Harvinder K. and Winnie Chee SS. Diabetes Care in Malaysia: Problem, New models, and Solution. Annals of Global Health.2015; 81(6):851-862.

59. Al-Goblan AS, Al-Alfi MA, Khan MZ. Mechanism linking diabetes mellitus and obesity. Diabetes Metab Syndr Obes. 2014;7:587-591. doi:10.2147/DMSO.S67400

60. Institute for Public Health (IPH) 2015. National Health and Morbidity Survey 2015 (NHMS 2015). Vol. II: Non-Communicable Diseases, Risk Factors \& Other Health Problems; 2015.

61. Güçel F, Bahar B, Demirtas C, Mit S, Cevik C. Influence of acupuncture on leptin, ghrelin, insulin and cholecystokinin in obese women: a randomised, sham-controlled preliminary trial. Acupunct Med. 2012;30(3):203-207. doi:10.1136/acupmed-2012-010127

62. International Diabetes Federation. IDF Diabetes Atlas. 8th Edition. Brussels: International Diabetes Federation, 2017. https://diabetesatlas.org/upload/resources/previous/files/8/IDF_DA_8e-ENfinal.pdf. Accessed 12 July 2021.

63. Birch S, Alraek T and Norheim AJ. The Journal of Alternative and Complementary Medicine. 2013;19(10): 845-850. DOI: 10.1089/acm.2012.0639.

64. Ratcliffe J, Thomas KJ, MacPherson H, Brazier J. A randomised controlled trial of acupuncture care for persistent low back pain: cost effectiveness analysis. BMJ. 2006;333:626, doi:10.1136/bmj.38932.806134.7C.

65. Willich SN, Reinhold T, Selim D, Jena S, Brinkhaus B, Witt CM. Cost-effectiveness of acupuncture treatment in patients with chronic neck pain. Pain. 2006 Nov;125(1-2):107-13. doi: 10.1016/j.pain.2006.06.006. Epub 2006 Jul 13. PMID: 16842918.

66. Nicolian S, Butel T, Gambotti L, Durand M, Filipovic-Pierucci A, Mallet A, Kone M, Durand-Zaleski I, Dommergues M. Cost-effectiveness of acupuncture versus standard care for pelvic and low back pain in pregnancy: A randomized controlled trial. PLoS One. 2019 Apr 22;14(4):e0214195. doi: 10.1371/journal.pone.0214195. PMID: 31009470; PMCID: PMC6476478. 
67. MacPherson H, Hammerschlag R, Lewith G, Schnyer R. (2008). Acupuncture Research. Strategies for Establishing an Evidence Base.

68. Ingle PV, Samdani NR, Patil PH, Pardeshi MS, Surana SJ. Application of acupuncture therapy in type 2 diabetes mellitus patients. Int J Pharm Sci. 2011;1:18-26.

69. He WJ, Zhao X, Li YQ et al. Adverse events following acupuncture: A systematic review of the Chinese literature for the years 1956-2010. J Altern Complement Med. 2012; 18:892-901.

\section{Table}

Table 1

Schedule for data collection*

\begin{tabular}{|llccc|}
\hline No & Measurement & Baseline & Week 3/4 & Week 7 \\
\hline 1. & Socio demographic information & $\sqrt{ }$ & \\
\hline 2. & $\begin{array}{l}\text { Anthropometry assessment } \\
\text { i. Weight } \\
\text { ii. Body mass index }\end{array}$ & $\sqrt{ }$ & $\sqrt{ }$ \\
iii. Waist circumference & & \\
3. & $\begin{array}{l}\text { Biochemical assessment } \\
\text { i. Fasting plasma glucose test }\end{array}$ & $\sqrt{ }$ & \\
& $\begin{array}{l}\text { ii. Fasting serum insulin test } \\
\text { iii. Homeostasis model assessment-insulin resistance }\end{array}$ & & $\sqrt{ }$ \\
\hline 4. & Health-related quality of life assessment & $\sqrt{ }$ & $\sqrt{ }$ \\
\hline 5. & Adverse event assessment & & $\sqrt{ }$ \\
\hline
\end{tabular}

*7 weeks of study period -6 weeks of treatment and 1 week of follow-up for adverse event after treatment ends.

\section{Supplementary Files}

This is a list of supplementary files associated with this preprint. Click to download.

- AdditioanalFile1.pdf

- AppendixIICRF.pdf

- AppendixIIIScreeningForm.pdf

- AppendixIVSamplepatientinformedconsent.pdf

- AppendixVStudybrochures.docx 
- AppendixVIStudyinformationsheet.pdf

- SPIRIT2013Checklist.pdf 